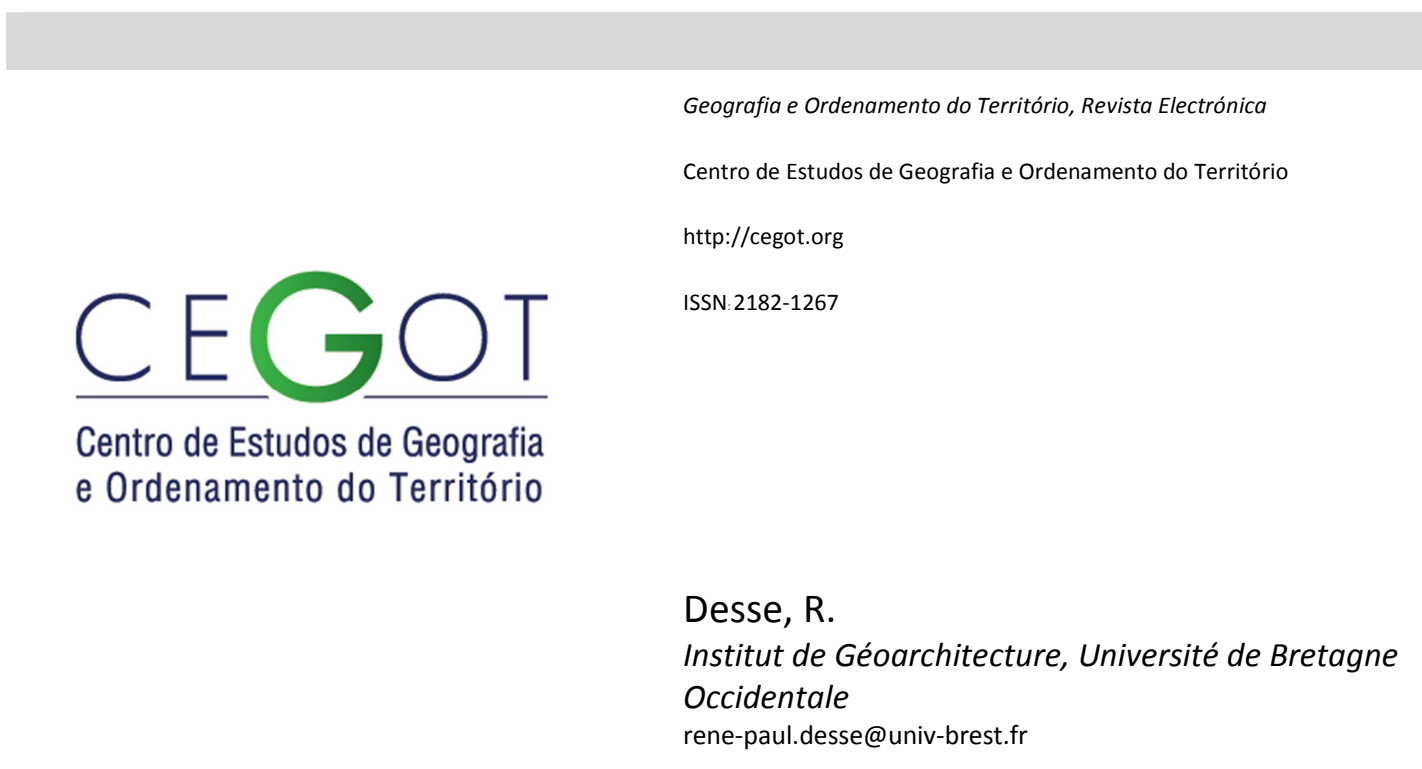

\title{
Quelle résilience pour les espaces commerciaux? Le cas français
}

Referência: Desse, R. (2014). Quelle résilience pour les espaces commerciaux? Le cas français. Revista de Geografia e Ordenamento do Território (GOT), n.o 6 (dezembro). Centro de Estudos de Geografia e Ordenamento do Território, p. 45-69

\section{Résumé}

La résilience peut être comprise comme la capacité d'un système à conserver ses structures principales en cas de fortes perturbations. Dans le cas du commerce, une forte concurrence et/ou une transformation de la demande par les consommateurs peuvent constituer ces changements auxquels sont confrontés les commerçants d'un espace donné : centre-ville, péricentral, périphéries. Les modifications du système marchand et du système urbain s'additionnent et complexifient l'analyse de la résilience commerciale, ce qui rend illusoire la tentative de modéliser la résilience d'un appareil commercial d'une ville dans sa globalité. Par contre, elle peut être utile à la compréhension des transformations d'un territoire urbain en particulier. La résilience comme outil stratégique est un champ de recherche à creuser dans le domaine des espaces commerciaux.

Mots-clés: Résilience commerciale, résilience des espaces commerciaux, résilience de la grande distribution.

\section{Resumo}

A resiliência pode ser entendida como a capacidade que um sistema tem de conservar as suas estruturas principais perante perturbações. No caso do comércio, uma concorrência forte e/ou a transformação do comportamento dos consumidores podem constituir 
perturbações com as quais os comerciantes são confrontados, num determinado espaço: centro de cidade, pericentral, periferias. As modificações do sistema comercial e do sistema podem somar-se a estas, dificultando a análise da resiliência comercial. Além disso, a complexidade dos fenómenos torna ilusória qualquer tentativa de modelizar a resiliência do aparelho comercial de uma cidade, vista na sua globalidade. No entanto, ela pode ser útil à compreensão das transformações dum determino território urbano, além do que a resiliência, como instrumento estratégico, é em si mesmo um campo de pesquisa a aprofundar no domínio dos espaços comerciais.

Palavras-chave: Resiliência comercial, resiliência dos espaços comerciais, resiliência da grande distribuição

\section{Introduction}

Comment se fait-il qu'une même transformation de la ville et des habitudes des consommateurs puisse être vécue par certains commerçants comme un choc, une catastrophe, et par d'autres comme un événement qui les fera évoluer vers d'autres formes commerciales ? Par "catastrophe ", nous envisageons un phénomène ou un ensemble de phénomènes dont les effets sont dommageables (Dauphiné, 2007). Ainsi, I'arrivée de nouvelles formes de distribution, à la charnière des années 1950 et 1960, a pu être considérée comme un événement catastrophique par beaucoup de commerçants de l'époque. Face à une nouvelle concurrence de formats commerciaux inédits comme les supermarchés et les hypermarchés à la périphérie des villes, certains commerçants de quartier ou de centre-ville ont fait faillite, ont vu leur chiffre d'affaires péricliter et ont tenté d'atteindre l'âge de la retraite pour partir, ou ont su s'adapter aux nouvelles conditions de commercialité ${ }^{1}$. La réponse à ce différentiel de comportements face aux changements tient dans un mot, celui de résilience.

Couramment accepté et utilisé aux États-Unis, ce mot n'a été introduit que tardivement en Europe. En France, il a plus de difficulté à s'imposer. A la différence des pays anglo-saxons où la résilience est considérée comme un concept, dans l'Hexagone, elle ne constitue encore qu'une notion floue et incertaine (Cormier et alii, 2010). Puisant aux sources du

\footnotetext{
${ }^{1}$ Les modifications de facteurs locaux de commercialité concernent les évolutions qui ont eu une incidence significative sur le commerce considéré : changements concernant la clientèle, l'accessibilité, l'ouverture d'une locomotive commerciale à proximité comme une grande surface ou un centre commercial intégré au centreville, etc (Desse, 2008).
} 
concept de résilience, en psychiatrie ou en neuropsychologie, la plupart des urbanistes nord-américains, pionniers en la matière, ont mis l'accent sur son association avec la notion de post-disaster. Ainsi Brian Ladd l'utilise pour montrer le différentiel des réactions des Berlinois face à la destruction presque totale de la ville en $1945^{2}$. Plus tard, les géographes français retravailleront la notion en l'appliquant au domaine des risques (Dauphiné, 2007). De façon intrinsèque, l'analyse de la résilience intègre l'idée que le dommage occasionné par un ensemble de changements n'est pas lié à une relation entre deux facteurs mais à un ensemble de facteurs en interaction. A ce stade de la réflexion, nous pourrions reprendre à notre compte ce que précisait pour les systèmes spatiaux en général, Christina AschanLeygonie : "étudier la résilience d'un système spatiaux revient à centrer le travail sur le rôle des structures, des interactions spatiales et de l'environnement dans le fonctionnement du système lorsqu'il est affecté par une perturbation " (Aschan-Leygonie, 2000). De ce fait, la résilience peut être comprise comme la capacité d'un système à conserver ses structures principales en cas de fortes perturbations. Dans le cas du commerce, une forte concurrence et/ou une transformation de la demande par les consommateurs peuvent constituer ces changements auxquels sont confrontés les commerçants d'un espace donné (centre-ville, péricentral, périphéries). Les modifications du système marchand (nouvelles offres, nouvelles demandes) et du système urbain (changements sociodémographiques du quartier, nouvelles accessibilités par une rocade ou un transport en commun efficace, implantations de nouvelles activités) s'additionnent et complexifient l'analyse de la résilience commerciale (Barata Salgueiro, 2011).

Dans ces conditions, la résilience devient un élément de la mesure de la vulnérabilité et des ressources (Briguglio et alii, 2009) : mesure du potentiel d'ajustement du commerçant mais aussi, mesure de la capacité à rebondir et à s'adapter des différents espaces commerciaux. La résilience se situe donc au cœur d'une compréhension globale du système commercial, de ses multiples acteurs que sont les consommateurs, les commerçants, les acteurs

\footnotetext{
2 II semble intéressant de confronter cette analyse des espaces commerciaux à celles réalisées par des spécialistes des sciences politiques ou de la Défense nationale sur des populations civiles qui seraient confrontées à une agression extérieure qui pourrait prendre des formes multiples comme une pénurie d'électricité, une propagande ennemie introduite dans les médias ou un bombardement. Le Livre Blanc sur la Défense et la Sécurité Nationale fait apparaître pour la première fois cette notion de résilience. Mais des auteurs comme Henrotin avec le concept de résilience construite ou Cormier ont été bien plus loin dans la réflexion.
} 
institutionnels, sans oublier le support de cette activité, les espaces urbains en pleine transformation. La complexité des phénomènes rend illusoire la tentative de modéliser la résilience d'un appareil commercial d'une ville dans sa globalité. Par contre, elle peut être utile à la compréhension des transformations d'un territoire urbain en particulier (Soumagne, 2014).

\section{Le nouveau commerce périphérique perçu comme la «catastrophe originelle»}

\section{A - Constats: Chocs de la révolution commerciale des cinquante dernières années (1963-2013) et résilience commerciale :}

L'émergence puis la montée en puissance du commerce périphérique constitue l'élément majeur de l'évolution commerciale de ces dernières décennies. En quelques années, on est passé du modèle d'urbanisme commercial de rue qui s'intègre de longue date au tissu urbain, à celui du modèle du centre commercial, conçu comme un ensemble cohérent, fermé sur lui-même, puis au modèle des boîtes, synonymes de paysages hétéroclites, d'architectures approximatives. Tout récemment, les nouveaux parcs commerciaux ou retail parks ont redonné un peu d'urbanité à ces périphéries commerciales.

\section{Le bouclage de la périphérie}

Jusqu'au milieu des années 1960, l'essentiel de l'activité commerciale des villes françaises s'inscrit dans l'espace du centre-ville actuel. L'espace commercial traditionnel va subir de plein fouet le déferlement de ces nouvelles formes commerciales dans les périphéries qui se constituent à la même époque. Les parts de marché de l'espace central ne cessent de s'effriter face à l'agressivité des supermarchés, hypermarchés et des grandes et moyennes surfaces spécialisées. A un centre-ville, creuset du lien social, unique pôle de la cité reconnu par tous, se substitue un espace morcelé, archipel de pôles commerciaux, fait de pratiques à la carte, selon le temps dont on dispose, du niveau de revenu, du degré de mobilité.

La première vague d'innovations survient au lendemain de la seconde guerre mondiale, à la fin de la période de pénurie, notamment par le biais du discount. Très vite s'ajoutent de 
nouvelles formes de la distribution : le libre-service et le supermarché, puis l'hypermarché ${ }^{3}$. L'innovation, cette fois-ci, est bien américaine. Souvent néophytes dans le commerce, les fondateurs des grands groupes français font le voyage aux États-Unis, qui ont quelques longueurs d'avance tant sur le plan de la motorisation des ménages que des formes de distribution. En 1963, le premier hypermarché français ouvre à Sainte-Geneviève-des-Bois en région parisienne. Marcel Fournier et Louis Defforey innovent et montrent la voie, en ouvrant le premier hypermarché Carrefour dans un tissu résidentiel de lotissements de l'entre-deux-guerres pour classes moyennes en phase de motorisation. Dès l'ouverture, cette grande surface, dite " alimentaire ", regroupe déjà tout " sous le même toit " $60 \%$ de la superficie est consacré au textile, à l'équipement de la maison, au bazar). À partir de la fin des années 1960, le modèle est reproduit un peu partout en France. Dès les premières implantations, les hypermarchés se localisent sur les communes périphériques des grandes agglomérations, au gré des opportunités foncières, selon la géographie des pénétrantes et des tracés hypothétiques de la future rocade. Depuis les années 1990, le mouvement d'expansion est en pleine décélération. Les grands groupes de la distribution et certains hommes politiques, incriminent les différentes réglementations qui freinent leur évolution et empêchent les nouvelles ouvertures. En réalité, plusieurs études montrent que le marché français est saturé et qu'avec 2050 hypermarchés en fin 2014, le bouclage des périphéries est achevé pour reprendre l'expression utilisée par Alain Metton dès 1998.

L'importation en France du Regional Shopping Center américain date de la fin des années 1960. Les urbanistes et aménageurs français le baptisent, très vite, centre commercial régional. En Région parisienne, de 1969 à 1974, une ceinture d'une quinzaine de ces centres se localise en banlieue. II s'agit d'opérations planifiées faisant appel à de grands promoteurs commerciaux (Belle-Epine, Créteil-Soleil, Les Quatre Temps à La Défense...). Puis le modèle se diffuse dans les grandes villes de province avec un certain nombre de variantes. La périphérie s'organise d'abord autour des hypermarchés, premier équipement des banlieues. La deuxième étape consiste à transformer ces implantations isolées en véritables centres commerciaux périphériques. Le nombre de petits commerces y varie de vingt à soixante

\footnotetext{
${ }^{3}$ Un supermarché est une surface de vente de 400 à $2500 \mathrm{~m}^{2}$. L'hypermarché est un néologisme français créé par une revue française de la grande distribution, Libre Service Actualité, après l'ouverture du premier Carrefour en France en 1963. Sa surface de vente dépasse les $2500 \mathrm{~m}^{2}$.
} 
unités. Dans les années 2000, la montée en gamme est réelle. Les succursalistes et franchisés des enseignes nationales se doivent d'être dans l'hypercentre, mais aussi dans les deux ou trois premiers centres périphériques selon la taille de l'agglomération. Des enseignes traditionnellement de centre-ville comme la FNAC, Zara ou H\&M multiplient les ouvertures dans des centres commerciaux de la périphérie. La crainte est perceptible, dans le milieu commerçant, de voir s'organiser en périphérie une véritable concurrence à l'offre haut de gamme du cœur de la cité ${ }^{4}$.

A l'exception des hypermarchés et des centres commerciaux, ce mouvement d'exurbanisation du commerce reste un phénomène limité jusqu'aux années 1980. A partir des années 1985, 1986, les Grandes et Moyennes Surfaces Spécialisées (GMSS comme Darty ou Decathlon) se multiplient en s'agglutinant la plupart du temps à proximité des premiers venus. Comme pour les hypermarchés, la diversification se poursuit, année après année, le secteur de l'équipement de la personne puis du domaine des loisirs et de la culture venant diversifier l'offre commerciale de ces "routes du meuble ». Dans les années 2000, les opticiens sont les derniers venus après le jouet, le livre... Autant de parts de marchés transférées du centre vers la périphérie.

L'offre des zones d'activités commerciales ne cesse d'évoluer, jouant sur l'effet de masse et de la diversification pour renforcer leur attractivité. Depuis les années 1990, chaque grand pôle périphérique s'accompagne de l'implantation de la restauration rapide, comme McDonald's ou Quick, ou des chaînes de restauration plus classique (Campanile, Courtepaille) ou à thème (Buffalo Grill, El Rancho). Le premier pas est franchi d'une pratique non commerciale de ces espaces, ensuite accentuée avec l'arrivée des multiplexes. Né aux États-Unis, ce nouveau phénomène pénètre dans I'Union européenne, d'abord en Belgique et en Grande-Bretagne, puis en Allemagne et aux Pays-Bas. En 1993, cet équipement de loisirs arrive en France. En début 2014, 190 multiplexes, dont la moitié en périphérie, totalisent $60 \%$ de la fréquentation des cinémas français.

Dans les réalisations commerciales les plus récentes et les nombreux projets en Europe, l'intégration des loisirs devient monnaie courante. À l'instar de ce qui se passe depuis

\footnotetext{
${ }^{4}$ En début 2014, 750 centres commerciaux se partagent $25 \%$ des parts de marché du commerce de détail. Les quatre cinquièmes se situent en périphérie.
} 
plusieurs années aux États-Unis, des centres commerciaux élargissent leurs offres. Fortement valorisés dans notre société, les loisirs ont tendance à marquer les zones commerciales où ils se localisent d'une image fortement positive. Ces nouvelles centralités alliant réellement les loisirs et les commerces sont encore assez rares en France : Bercy Village à Paris, Odysseum à Montpellier semblent inaugurer de nouveaux modes de consommation ${ }^{5}$. En important en périphérie, d'authentiques éléments du centre-ville, comme les loisirs, les acteurs de l'aménagement (promoteurs, grands groupes de la distribution, collectivités locales) contribuent à renforcer l'attractivité de certains espaces commerciaux.

Toutes ces années de développement du commerce périphérique engendrent une nouvelle organisation de l'appareil commercial des villes françaises. Son poids en termes de parts de marché est devenu plus important que celui de l'espace central. Celui-ci ne constitue plus qu'une des principales centralités commerciales de la cité, dans un système multipolaire de plus en plus complexe. Quant à la périphérie conquérante, elle ne se présente pas de façon monolithique. Selon la taille des agglomérations, un ou plusieurs pôles principaux se partagent l'essentiel de la clientèle. Le dynamisme commercial n'est pas partout identique.

\section{B - Quelle résilience dans ces espaces périphériques en 2014 ?}

Depuis l'ouverture du premier hypermarché en 1963, l'émergence d'un nouveau tissu commercial est tellement radicale et brutale qu'elle peut être assimilée à un choc voire pour certains commerçants à une "catastrophe ", notamment ceux des espaces marchands «traditionnels» du centre-ville ou de la proximité. Quant à la périphérie elle-même, l'équipement commercial semble achevé, voire par endroit atteindre les limites de la saturation. Les premières "catastrophes " arrivent, sous la forme de l'installation d'un concurrent, un category killer pour les spécialistes du marketing. Dans ces conditions, la résilience peut être envisagée comme la capacité de résister à ce mouvement dévastateur, et de se reconstruire en faisant évoluer sa forme commerciale. Comme dans la notion de résilience développée par les psychologues, il semble que cette capacité à résister et à

\footnotetext{
${ }^{5}$ Les ouvertures des grandes surfaces de l'opération Odysseum se sont échelonnées de 2005 à 2009. L'ensemble propose en 2014, multiplexe, patinoire, planétarium, une trentaine de restaurants et $120000 \mathrm{~m}^{2}$ de surfaces commerciales, le tout connecté au reste de l'agglomération de Montpellier par le tramway.
} 
rebondir prenne appui sur deux facteurs primordiaux : le rôle de la personnalité du manager et de l'environnement commercial et urbain. Concernant le premier facteur, se pose la question de la vulnérabilité et des ressources du commerçant. Dans ces espaces périphériques, les investigations sont quasi impossibles, les "pères fondateurs " d'un nouveau concept commercial ayant transformé leur "aventure personnelle » en une " histoire » d'un groupement d'indépendants (Leclerc, Intermarché, Super U) ou d'un groupe intégré (Carrefour, Casino, Auchan, etc.). Il semble donc que cette résilience soit à étudier au moyen d'une grille d'analyse du domaine de la théorie des organisations, voire du monde de la finance et de la bourse.

\section{Les degrés de résilience de la grande distribution.}

Dans ces espaces périphériques, les " inventeurs du commerce moderne " ont fait émerger les nouvelles formes du commerce (Thill, 1966). A la différence de ce qui s'est passé dans le secteur industriel, les innovations sont rarement venues des grands groupes existants comme le Printemps, les Galeries Lafayette qui avaient su développer la formule du grand magasin à la fin du XIX ${ }^{e}$ siècle puis celle des magasins populaires après la crise de 1929 . Une enseigne comme Félix Potin, qui développe un réseau de magasins d'alimentation dès le XIX siècle, arrive à son maximum en 1956 avec 1200 unités, avant de péricliter et de disparaître complètement en 1996. Ses dirigeants n'ont pas su évoluer et prendre le tournant de surfaces alimentaires plus importantes, faisant montre ainsi d'une faible résilience (capacité à survivre quelques décennies au choc initial).

Paradoxalement dans les années 1960, l'innovation vient souvent de commerçants sortis du rang, des commerçants bien installés dans leurs villes d'origine comme Marcel Fournier et Louis Defforey en Savoie (Carrefour), Paul-Auguste Halley à Caen (Promodès) ou la famille Darty en Région parisienne. Quelques innovateurs proviennent d'horizons très éloignés du commerce comme la famille Mulliez de Roubaix (famille d'industriels qui est à l'origine d'Auchan) ou Edouard Leclerc, à Landerneau qui donnera son nom au premier groupe coopératif de la grande distribution française. Cette histoire de l'émergence de la grande distribution reste à faire. Les historiens du commerce ont amorcé l'étude sans donner les clés de lecture de ces aventures individuelles: comment ainsi expliquer l'évolution différente entre deux frères issus d'une famille non commerçante, l'un restant épicier 
"traditionnel », l'autre à la tête d'un des premiers groupements de grandes surfaces alimentaires françaises à envergure internationale ${ }^{6}$ ? Sans doute, les investigations à mener, les grilles d'analyses à réaliser pour comprendre cette réaction face au changement sont plus du ressort de la psychosociologie que du domaine du marketing.

Soixante ans après l'ouverture du premier hypermarché français, force est de constater un grand dynamisme du secteur de la grande distribution, lui permettant de s'adapter aux transformations de la société et aux nouvelles attentes des consommateurs. Ici ou là des enseignes ont disparu, des groupes ont été absorbés par d'autres mais globalement cette forme de distribution a montré sa forte résilience. Ce dynamisme provient en partie de la capacité d'attirer les capitaux. Si dans les premiers temps, l'autofinancement et les emprunts nécessaires auprès des banques n'ont été possibles que par des bénéfices importants, peu à peu le secteur se financiarise, attirant des investisseurs de plus en plus étrangers au commerce. Ainsi, dans les années 1990, les sociétés d'hypermarchés ont connu de profondes modifications de leurs assises financières. Les implantations devenant plus difficiles, le marché commençant à saturer, les achats-fusions se multiplient via des opérations publiques d'achat. Dans ce secteur ne subsistent actuellement que six grandes entreprises: Leclerc, Carrefour, Intermarché, Super U, Auchan et Casino qui se partagent l'essentiel de l'activité et quadrillent le territoire, réduisant de fait la concurrence. A partir des années 2007-2008, la bulle immobilière ayant éclaté, les investisseurs se reportent sur les projets commerciaux (Mérenne-Schoumaker, 2014). La financiarisation de l'immobilier commercial s'accélère en plein ralentissement de la consommation. Le commerce est rentré dans sa bulle pour reprendre l'expression de Pascal Madry, à quand l'éclatement ? (Madry, 2011).

Dernier facteur de grande résilience, dans le domaine de l'assise financière, la plupart de ces groupes de la grande distribution poursuivent leur expansion hors de l'Hexagone. Carrefour occupe la deuxième place, après le groupe américain Wal-Mart, dans la hiérarchie mondiale réalisant $60 \%$ de son chiffre d'affaires à l'étranger (Chine, Amérique latine), ce qui lui permet d'affronter quelques difficultés sur le marché français.

\footnotetext{
${ }^{6}$ Des deux frères Leclerc, I'un Paul Leclerc a pris sa retraite d'épicier à Landerneau, dans les années 1990, l'aîné Edouard Leclerc a donné son nom aux hypermarchés et supermarchés du groupement.
} 
Face à la concurrence des autres enseignes, la plupart de ces grands groupes ont pour l'instant des marges financières suffisantes pour évoluer. II en va ainsi des hypermarchés dont certains auteurs ont tenté de démontrer la mise à mal de ces formats holistiques pour reprendre la formule de Philippe Moati (Moati, 2011). Depuis 2008, la plupart de ces enseignes ont amorcé une nouvelle révolution commerciale en multipliant les drives prenant ainsi en marche la révolution d'internet en utilisant un de leurs principaux acquis, une logistique sans faille. Pourtant quelques grands groupes semblent présenter une résilience plus faible, face aux évolutions récentes (crise économique et progression du commerce électronique). Ainsi, le groupe Auchan voit son chiffres d'affaires en repli pour 2013, y compris dans son bastion du Nord de la France $(-2,5 \%)$ et dans ses nouvelles implantations russes.

\section{Les degrés de résilience des espaces commerciaux périphériques.}

(Tableau final de synthèse: Degré de résilience dans les espaces commerciaux des villes françaises, Type 4 résilience commerciale très forte (RC4).

Depuis les années 1990, les premières tensions apparaissent en périphérie sans qu'il y ait vraiment d'événements «catastrophiques» comme la fermeture de l'ensemble des magasins d'une zone d'activités commerciales. Les friches commerciales peuvent apparaître; elles sont en général d'ampleur limitée comme dans les zones urbaines sensibles ou affectant des nodules commerciaux de petite taille qui, pour une raison ou une autre, perdent leurs locomotives commerciales (hypermarchés) et qui de ce fait même sont moins attractifs.

En périphérie, si les enseignes bougent beaucoup dans certains secteurs, globalement les espaces commerciaux sont assez stables. Les modifications du dynamisme commercial de tel ou tel de ces pôles périphériques sont possibles mais limitées par un degré de résilience très élevé. Les acteurs principaux que sont les grands groupes de la distribution ne peuvent se permettre, dans la plupart des cas, de perdre des investissements importants par la fermeture d'une unité commerciale, fermeture qui nuirait à l'image de marque de l'enseigne, alors que toutes les campagnes publicitaires tentent de montrer le dynamisme de l'entreprise. Au pire, si une enseigne comme Toy'R'us disparaît en partie du paysage commercial français, ses implantations privilégiées, sur les meilleurs emplacements dans les 
zones d'activités commerciales des grandes villes, permettront de le remplacer aisément. Les lois du marché immobilier viendront « réparer » une erreur de marketing ${ }^{7}$.

Mais si ces espaces périphériques sont nés de l'initiative de nouveaux commerçants, ceux-ci ont su anticiper les évolutions de la ville voire les renforcer. Remarquons que le commerce périphérique se localise à proximité des rocades autoroutières et souvent de pénétrantes, profitant ainsi de flux motorisés très importants. Proches de ces implantations commerciales en intra-urbain, les grands ensembles d'habitat collectif concentrent des populations nombreuses, mais au pouvoir d'achat moyen voire faible. Au contact de la rocade commencent les vastes quartiers périphériques puis périurbains qui fournissent de faibles densités de population, mais à pouvoir d'achat plus élevé. Dans le cadre d'une forte mobilité en voiture, le «nouveau » commerce a une accessibilité bien plus grande que les magasins de centre-ville. Pendant longtemps délaissés par les collectivités locales, ce n'est que tout récemment qu'elles s'y sont intéressées en aménageant les espaces publics ou en les connectant au réseau de transport collectif en site propre comme le tramway (Soumagne, 2011).

Pour l'instant, on peut se poser la question de la pertinence de la notion de résilience pour les périphéries et leurs principaux acteurs, les grands distributeurs. II ne peut avoir de résilience qu'après une "catastrophe ", un choc commercial. Or, il y a une telle capacité à évoluer, à anticiper, à réagir aux moindres signes de difficultés sans attendre l'effet déflagrateur, que les exemples d'effondrements en périphérie sont encore extrêmement rares. Dans les années qui viennent, il se pourrait qu'avec la saturation du marché et la progression exponentielle des projets, on assiste aux premières vraies difficultés.

\footnotetext{
7 L'échec de Toy'R'us en France s'explique par la saisonnalité des achats de jouets : plus de 50\% d'entre eux s'effectuent pour noël dans l'Hexagone, ce qui n'est pas le cas dans les pays anglo-saxons où la majorité des ventes s'éparpillent toute l'année au gré des anniversaires. Dans le cas français se pose le problème du coût de l'emploi et des stocks durant une grande partie de l'année.
} 


\section{Un espace globalement de faible résilience: le centre-ville commerçant ?}

\section{A - Les trois phases de la résilience commerciale}

L'analyse du tissu commercial de centre-ville permet de développer des investigations plus en profondeur sur cette notion de résilience que nous pouvons décliner en trois phases.

- Première phase: situation «initiale» des années 1950 et 1960 où le "petit » commerce semble vivre son âge d'or, du moins si l'on ne considère que les effectifs. À la veille de la modernisation de l'appareil de distribution avec l'arrivée des hypermarchés de périphérie, le centre-ville, pour un grand nombre d'achats, reste sans grand concurrent. Grands magasins, magasins populaires et commerces rares en constituent les élémentsphares. Dans les villes moyennes françaises, à la fin des années 1960, on peut estimer approximativement que l'appareil commercial central constitue entre 70 et $80 \%$ du chiffre d'affaires de l'agglomération (le restant correspondant au commerce de proximité).

- Deuxième phase: La "Catastrophe» ou l'émergence de la grande distribution. Le quadrillage des villes françaises par les supermarchés et les hypermarchés n'est effectif que dans les années 1970, voire 1980 pour les petites villes. Le nombre des commerces s'effondre, notamment dans les espaces péricentraux. Les tensions sont fortes, s'exprimant parfois de façons violentes : ainsi, à partir de 1969, Gérard Nicoud, un syndicaliste virulent, multiplie les actions d'éclat luttant d'une part « contre l'État qui asphyxie le petit commerce par les hausses des cotisations sociales et d'autre part contre la grande distribution " en perturbant les inaugurations de grandes surfaces. Son action syndicale est sans doute la dernière d'un monde qui s'achève. II n'est pas sûr que les commerçants qui ont suivi le CIDUNATI aient su anticiper les changements, plus focalisés sur les moyens de lutter contre un ennemi insaisissable que sur les transformations de leur propre commerce.

- $\quad$ Troisième phase: Situation que les spécialistes de la résilience qualifient de «retour à la vie», celle-ci étant rarement un retour à l'état antérieur mais plutôt l'émergence d'une situation nouvelle. Ainsi, cet espace central marchand, une quarantaine d'années plus tard, ne représente plus que 20 à $30 \%$ du chiffre d'affaires global, la proximité (commerce de quartier) grossièrement $10 \%$, et le nouveau venu, le commerce périphérique, 60 à $70 \%$. 
Dans cette troisième étape, la résilience peut être envisagée comme la capacité de résister à ce mouvement dévastateur que certains pourront assimiler à un traumatisme, et de se reconstruire après lui en faisant évoluer sa forme commerciale.

Se pose alors la question de la vulnérabilité du commerçant aux changements de son secteur et de la société toute entière. Comment mesurer la résilience de ce dernier, sachant que deux commerçants, dans la même rue, ayant donc un environnement semblable, ne réagiront pas de la même manière ? Sans doute, les investigations à mener pour comprendre ces réactions face au changement ne sont pas exclusivement du ressort du marketing et de l'urbanisme. Quant au rôle de l'environnement commercial et ses transformations dans la résilience finale, il constitue l'objet même de cette étude.

\section{B - Degrés de résilience des espaces commerciaux centraux}

Les transformations du commerce des centres-villes françaises vont accentuer les différenciations entre les divers espaces centraux et les commerçants eux-mêmes qui en constituent les principaux acteurs. Dans le cadre d'une " évolution post-catastrophe ", les processus hypothétique de résilience pour arriver à un nouveau stade d'évolution (stade 2) intègrent les ressources internes et externes de ces espaces centraux.

Dans l'inventaire de ces ressources internes, nous intégrons les changements dans l'environnement immédiat susceptibles de venir renforcer certains espaces marchands du centre-ville. Ainsi, cet espace a été fortement perturbé par le déclin démographique, notamment lié au départ des familles des espaces centraux. Depuis deux décennies, la mise en œuvre des politiques de rénovation, restauration et de réhabilitation a permis d'enrayer le départ des populations résidentes puis d'en attirer d'autres. Ces nouvelles clientèles potentielles ont renouvelé les problématiques du commerce de proximité en permettant à la grande distribution de déployer de nouveaux formats comme Carrefour City, Casino Shop ou U Express; ou pour les quartiers gentrifiés, Monop' ou Chez Jean. L'alimentation traditionnelle n'a pas survécu à la concurrence des grandes surfaces périphériques et au départ de ses clients.

La morphologie des quartiers centraux a pu jouer en faveur de l'appareil commercial. Ainsi, des tissus médiévaux, qui étaient voués à la destruction dans les années 1950, sont devenus 
des quartiers à forte valeur patrimoniale (Fernandes et alii, 2007), parfois valorisés dans le cadre de Secteurs sauvegardés. Leur mise en tourisme permet à ces centres d'attirer de nouvelles clientèles aux attentes spécifiques. Le développement des courts séjours a favorisé une fréquentation sur plusieurs mois de l'année et non plus seulement durant la saison estivale.

Dans les ressources des centres-villes comme forme collective de résilience, la constitution d'associations de commerçants peut être citée. Celles-ci se sont organisées pour animer le centre-ville, participer au montage d'un projet financé par le FISAC ${ }^{8}$ ou contrer une nouvelle opération susceptible de nuire aux espaces centraux. En France, ces associations ne regroupent qu'une faible partie des commerçants d'où une assise financière relativement modeste et un pouvoir d'interpellation des collectivités locales assez faible. En définitive, leur influence a été secondaire et quand elle a été réelle, elle est souvent venue des commerçants des espaces les plus dynamiques de l'hypercentre ${ }^{9}$.

Dans l'inventaire des ressources externes, nous intégrons les changements à l'échelle de la ville donc extérieurs aux centres-villes et les transformations liées à l'action des acteurs externes (acteurs privés et institutionnels).

Les évolutions récentes de ces espaces urbains en France ont été peu favorables au cœur de la cité. Parallèlement au mouvement d'exurbanisation du commerce, de nombreuses fonctions qui étaient le privilège du centre se sont relocalisées en périphérie (université, hôpital, centre administratif) à proximité des premières zones industrielles puis des parcs d'activités. Dès les années 1970, pour atténuer le déclin du centre-ville, les Municipalités amorcent une réflexion et mettent en œuvre les premières grandes opérations de restructuration. II s'agit, dans un premier temps, de mettre en place de simples mesures d'accompagnement, comme la création de rues piétonnes ou le renforcement du réseau de transports en commun. Puis, dans un deuxième temps, de concevoir de véritables

\footnotetext{
${ }^{8}$ Institué par la loi du 31 décembre 1989, le FISAC ou Fonds d'Intervention pour les Services, l'Artisanat et le Commerce est un dispositif d'aide à la régénération commerciale des territoires ruraux et urbains qualifiés de fragiles : campagnes en voie de dépeuplement, bourgs ruraux et centres de villes petites et moyennes, faubourgs et banlieues en difficulté.

${ }^{9}$ En reprenant la grille d'analyse utilisée par Teresa Barata Salgueiro en 1992 et reprise en 2011 dans une autre étude sur le centre-ville de Lisbonne, il serait utile de poursuivre dans cette voie en regroupant les commerçants en trois groupes plus ou moins résilients : groupe des commerçants passifs, réactifs ou proactifs (Barata Salgueiro , 1992 ; Guimarães, Matos., Barata Salgueiro, 2011).
} 
opérations d'urbanisme commercial, comme les centres commerciaux intégrés (La Part-Dieu à Lyon, Mériadeck à Bordeaux, Le Polygone à Montpellier, etc). Derrière cette nouvelle approche des problèmes, se manifeste la volonté des villes de remodeler leur tissu commercial.

Les politiques de piétonisation ou la réalisation d'un centre commercial intégré ont comme principale conséquence de renforcer l'attractivité de certains espaces et d'en marginaliser d'autres. Dès leur ouverture, ces nouvelles locomotives accélèrent les turbulences commerciales et modifient les stratégies d'implantation. La commercialité augmentant, les loyers suivent à la hausse. Le commerçant indépendant ne peut plus "faire face » et est remplacé par un franchisé ou un succursaliste. L'hypercentre se renforce au détriment du pourtour de l'espace central et de l'ensemble du péricentral. Dans les rues adjacentes à l'axe principal, les linéaires commerciaux s'étiolent, le commerce de détail recule. Ces rues connaissent un réel déclin commercial avec une augmentation notable des cellules vides et des services. Ces évolutions entraînent une spécialisation des espaces avec une concentration commerciale sur l'équipement de la personne et les loisirs.

Quelques constats généraux caractérisant ces espaces centraux au terme du processus de résilience:

- L'ensemble des espaces marchands du centre-ville a cessé de perdre des effectifs ;

- Les commerçants localisés sur les marges ont le plus souffert ;

- La rétraction des espaces marchands dynamiques a suscité un morcellement de I'hypercentre autour d'un secteur piétonnier, d'un centre commercial intégré ou d'une locomotive commerciale isolée de type FNAC;

- Le commerce indépendant a fortement reculé au profit des succursalistes et des franchisés.

En termes de processus de résilience, pour atteindre cette situation de stade 2, une combinatoire d'actions va expliquer ce différentiel de résilience selon les divers espaces centraux (Tableau final de synthèse : Degré de résilience dans les espaces commerciaux des villes françaises, Type 3 résilience commerciale forte (RC3): 
- Les acteurs institutionnels peuvent jouer sur la restructuration des espaces publics dont la voirie (piétonnisation, politiques de parking, de réduction de l'accessibilité automobile, développement des transports collectifs en site propre comme le tramway) ; sur des projets de centres commerciaux en ayant la maîtrise du foncier... Mais leurs actions s'arrêtent là, l'élément moteur, l'investissement privé étant nécessaire pour achever l'opération.

- Les acteurs privés, qu'ils s'agissent des foncières, des promoteurs commerciaux ou des grands distributeurs s'intéressent de plus en plus aux centres-villes, au fur et à mesure que les périphéries sont saturées.

- La spécificité de l'espace central, c'est l'obligation pour ces deux types d'acteurs de « travailler » ensemble, l'un ayant la maîtrise du sol (permis de construire, autorisation en Commission départementale d'aménagement commercial10), l'autre ayant l'assise financière et le savoir-faire qui assure la rentabilité de l'opération. Ce processus de résilience combinatoire concernant la revitalisation du commerce central pourrait être aussi qualifié de cogestion locale étroite entre acteurs privés et acteurs publics, au risque d’un mélange des genres (Desse, 2013).

- De ce processus de résilience qui va faire émerger un nouveau paysage commercial, le grand perdant est le commerçant indépendant qui n'a pas su ou pas pu évoluer face à la montée des baux commerciaux dans les espaces convoités, dans un contexte de concurrence de la grande distribution.

\section{Quelques espaces de forte résilience en péricentral ont tendance à s'organiser en centralités autonomes.}

Dans cette confrontation entre le centre-ville et les grandes surfaces périphériques, les espaces péricentraux ont pendant longtemps connu une très sévère diminution du nombre des petits commerces de recours quotidien. Avec un décalage de quelques années, voire

\footnotetext{
${ }^{10}$ Depuis 1973, en France, a été mis en place des dispositifs de régulation des ouvertures des grandes et moyennes surfaces commerciales. Des Commissions départementales et nationales d'aménagement commercial autorisent ou n'autorisent pas les ouvertures de ces magasins (Desse et alii, 2008). Actuellement les CDAC donnent leur aval à $85 \%$ des dossiers présentés.
} 
d'une décennie, son expansion avait suivi l'urbanisation des années cinquante et soixante. Présent sur les axes traditionnels de sortie de la ville mais aussi dans les grands ensembles collectifs, ce commerce péricentral a subi de plein fouet la concurrence des grandes surfaces périphériques. Au service d'une clientèle de quartier, il a souffert du déclin démographique de certains secteurs de la ville et de la motorisation de plus en plus poussée des ménages. Conçu pour une population de proximité, il s'adapte de plus en plus mal à une mobilité accrue de sa clientèle. De ce fait, la résilience de la plupart de ces quartiers est faible, le commerce a pratiquement disparu à quelques exceptions avec des centralités isolées, souvent récentes (Tableau final de synthèse: Degré de résilience dans les espaces commerciaux des villes françaises, Type 1 résilience commerciale faible et moyenne (RC1 + $\mathrm{RC2})$.

Paradoxalement, c'est aussi dans certains de ces quartiers péricentraux que les processus de résilience ont pu fonctionner, permettant l'émergence de nouvelles centralités aux formes commerciales totalement renouvelées. Ainsi, le commerce de transit se développe, préférant se localiser sur les parcours des consommateurs de plus en plus mobiles, que de s'implanter dans les espaces résidentiels (Desse, 2014). Le mouvement est déjà ancien dans les aéroports ou les gares comme la gare Saint-Lazare à Paris. Dans les villes de province, le phénomène est plus ponctuel: se localisent ainsi, sur des axes de circulation, des boulangeries, des fleuristes voire des pharmacies, parfois en remplacement d'une stationservice, ce qui permet l'usage d'un parking. Depuis peu, la grande distribution s'intéresse à ces niches commerciales localisées sur des pénétrantes ou des rocades, multipliant ses nouveaux petits formats. Sur ces axes majeurs de la mobilité urbaine, les hypermarchés amorcent depuis 2008-2009 un développement rapide des drives. Adossés à un hypermarché classique ou isolé, le drive permet de commander ses achats sur internet et de "passer » ensuite réceptionner les produits avec sa voiture, le plus souvent sur le trajet d'un déplacement lieu de travail-lieu de résidence. Simple entrepôt, cet hypermarché virtuel a besoin de moins de surface au sol, de parkings moins importants (le turn over des automobilistes étant très rapide $)^{11}$.

\footnotetext{
${ }^{11}$ Dans l'Hexagone, ils étaient 400 en début 2010, 1000 en début 2012, 3100 drives en mai 2014 (2100 drives solo ou entrepôts autonomes et éloignés d'un magasin; et 1000 services drive où le client se gare sur le parking du supermarché ou de l'hypermarché et retire la marchandise commandée via internet à l'accueil. Cet
} 
Les limites de la notion de résilience appliquée aux espaces commerciaux: l'exemple des waterfronts.

Après une période de relative accalmie, au début des années 1990, les réalisations de centres commerciaux intégrés à ces espaces péricentraux reprennent. Ainsi, depuis le début des années 2000, les opérations se multiplient sur les fronts d'eau ou waterfronts. Avec parfois des décennies de retard sur les opérations britanniques, ou portugaises ${ }^{12}$, la revitalisation des premiers bassins et des docks passe par la mixité fonctionnelle, le commerce et les loisirs constituant les locomotives comme à Bordeaux "le Quai des marques ", au Havre "les Docks Vauban » ou à Rouen "Docks 76 » (Gasnier, 2013). Le paradoxe vient du fait que ces espaces longtemps délaissés par l'activité portuaire n'ont jamais été des centralités commerciales importantes. Dans leur proximité immédiate, les quelques commerces du quartier ont peu à peu périclité, les derniers cafés ont fermé, au fur et à mesure du départ des habitants et de la baisse des effectifs des ouvriers du port.

La catastrophe constitutive de cet abandon, le départ de l'activité portuaire en aval à la recherche de meilleurs tirants d'eau et de zones de stockage plus vastes, n'a pas suscité d'évolution rapide. Quelques quarante à cinquante ans ont pu s'écouler avant qu'il y ait effectivement une nouvelle transformation. D'une faible résilience commerciale, on est passé à une forte résilience sans que ces espaces aient changé. Toujours délaissés, ils deviennent brutalement attractifs car repérés par les acteurs institutionnels (Collectivités locales, SEM, Port autonome) comme pouvant être le support de grosses opérations d'urbanisme commercial (seuls espaces libres à proximité du centre, coûts faibles du foncier, nouvelle problématique urbanistique de reconquête des rives du fleuve ou d'un fond de port). Choisis par les Institutionnels qui en assurent la maîtrise du foncier, ils deviennent attractifs pour les promoteurs commerciaux et la grande distribution. A ce stade, nous atteignons les limites de la notion de résilience et pouvons énoncer l'hypothèse suivante : tout espace, quel que soit son degré de résilience actuel, peut devenir le support

essor de ce nouveau mode d'approvisionnement est très "français". En Belgique, par exemple, le premier drive solo a ouvert début 2014. Aux USA, Wal-Mart a inauguré sa première unité en avril 2014.

12 Exemple du centre commercial Vasco de Gama sur les rives du Tage à Lisbonne, ouvert en 1999, un an après l'exposition universelle de 1998, sur le site de l'évènement. 
d'une centralité commerciale importante. Il suffit pour cela que les acteurs privés et publics investissent dans le lieu, l'effet-masse assurant le succès de l'opération.

\section{Conclusion}

Derrière les spécificités des différents espaces commerciaux de la ville, de grandes règles apparaissent qui semblent perdurer dans le temps. Après l'événement " catastrophe " qui a déclenché la révolution commerciale des années 1960 et 1970, c'est-à-dire le surgissement de la grande distribution et le déclin du commerce indépendant, le tissu commercial s'est déployé en périphérie et s'est fragmenté au gré d'une mobilité motorisée des consommateurs toujours plus grande. Dans ces conditions, à quelques exceptions près, les formes de commerce novatrices sont issues de la grande distribution, seule capable d'avoir les capacités financières et opérationnelles de ce changement.

Cette nouvelle forme d'organisation du commerce au sein de groupements coopératifs ou de sociétés succursalistes est récente, à peine vieille d'un demi siècle. L'analyse de la résilience de ces acteurs issus du monde du commerce des années 1960 n'est pas simple à réaliser, même a posteriori. Sans doute, faudrait-il d'autres investigations pour comprendre la transformation d'un petit commerçant en leader national voire international et les mécanismes de son ascension.

Si cette notion de résilience a ses propres limites pour la compréhension de l'évolution des commerçants, elle nous semble plus opérationnelle pour les espaces commerciaux. Utilisant une combinatoire de facteurs internes et externes pour évaluer les degrés de la résilience, il est possible d'envisager une résilience construite, que l'on peut travailler de façon à ce que, face à un incident, une "catastrophe" sous la forme d'une arrivée brutale de la concurrence, ces espaces commerciaux soient plus résilients. La résilience comme outil stratégique est un champ de recherche à creuser. Réalisée à l'échelle de l'espace français, cette amorce d'analyse aurait tout intérêt à se poursuivre à l'échelle européenne. Des comparaisons entre pays permettraient dans doute de mieux dégager les facteurs de résilience des espaces commerciaux, en limitant la part des spécificités locales dans la prise en compte de ces processus. 


\section{Bibliographie}

Aschan-Leygonie, C. (2000). Vers une analyse de la résilience des systèmes spatiaux, Espace géographique, tome $29, n^{\circ} 1$, p.64-77.

Barata-Salgueiro, T. (1992). A cidade em Portugal, uma Geografia Urbana. Edições Afrontamento, Porto.

Barata-Salgueiro, T. (2011). "The resilience of urban retail areas", In Barata-Salgueiro T., Cachinho, H. (Editeurs). Retail planning for the resilient city. Consumption and urban regeneration. Editions Universidade de Lisboa, Centro de Estudos Geográficos, Lisboa, p.19-44.

Berkes, F., Folke, C. (1998). Linking social and ecological systems. Management Practices and social mechanisms for building resilience. Cambridge Press, Cambridge.

Briguglio, L., Cordina, G., Farrugia, N., Vella, S. (2009). Economic Vulnerability and Resilience : concepts and measurements, Oxford Development Studies, Vol. 37, n³, p. 229-247.

Cormier, P.-Y., Ferran, A., Rastouil, C., Turner, I. (2010). Les principes de la résilience en France, Collection des Chercheurs militaires $n^{\circ} 40$, Editions de l'enseignement militaire supérieur, ministère de la Défense, Paris, 90 pages.

Dauphiné, A., Provitolo, D. (2007). La résilience: un concept pour la gestion des risques, Annales de Géographie, $n^{\circ} 654$, p. 115-125.

Desse, R-P., Fournié, A., Gasnier, A., Lemarchand, N., Metton, A., Soumagne, J. (dir.) (2008). Dictionnaire du Commerce et de l'Aménagement, Presses Universitaires de Rennes, Collection Espaces et territoires, Rennes, 370 pages.

Desse, R-P. (2013). La fin de l'urbanisme commercial à la française. Les Annales de la recherche urbaine $n^{\circ} 108$, p. 4-15.

Desse, R-P. (2014). "Le commerce de transit, entre hypermobilité et gestion du temps ", In Gasnier, A., Lemarchand, N. (dir.), Le commerce dans tous ses états. Espaces marchands et enjeux de société. Presses Universitaires de Rennes, Collection Espace et territoires, Rennes, p.129-137.

Fernandes, J A. Rio, Guillemot, L., Saldanha, L P., Soumagne, J. (2007). As cidades do Porto et de Angers. Apontamentos sobre os processos de metrolização, terciarização e revitalização urbana. Edições Afrontamento, Porto, 164 pages.

Gasnier, A. (2013). La fonction commerciale dans les politiques de renouvellement des fronts deau urbains à Bordeaux et à Saint-Nazaire. Les Annales de la recherche urbaine $n^{\circ} 108$, p. 83-95.

Guimaraes, P., Matos, F., Barata-Salgueiro, T. (2011). Os comerciantes como actores da resiliência das áreas comerciais. In Barata-Salgueiro T., Cachinho, H. H. (Editeurs), Retail planning for the resilient city. Consumption and urban regeneration. Editions Universidade de Lisboa, Centro de Estudos Geográficos, Lisboa, p.105-124.

Ladd, B. (1997). The Ghosts of Berlin: confronting German History in Urban Landscape, University of Chicago Press, 271 pages.

Madry, P. (2011). Le commerce est entré dans sa bulle, Études foncières, 151, p.12-16.

Mérenne-Schoumaker, B., Nappi-Choulet, I. (2014). «Commerce et opérateurs immobiliers : un marché en voie de financiarisation ", In Gasnier, A., Lemarchand, N. (dir.), Le commerce dans tous ses états. Espaces marchands et enjeux de société. Presses Universitaires de Rennes, Collection Espace et territoires, Rennes, p.197-204.

Metton, A. (1998). Espoirs et amertumes du commerce de centres-villes, Les Annales de la Recherche urbaine, $\mathrm{n}^{\circ} 78$, p.47-54.

Moati, Ph. (2011). La nouvelle révolution commerciale, Éditions Odile Jacob, Paris, 315 pages.

Soumagne, J., Desse, R.-P., Grellier, A. (2011). «Commercial Crise and resilience in French urban peripheries». In Barata-Salgueiro T., Cachinho, H. (Editeurs),). Retail planning for the resilient city. Consumption and urban regeneration. Editions Universidade de Lisboa, Centro de Estudos Geográficos, Lisboa, p.81-104. 
Soumagne, J.(dir.), Desse, R-P., Gasnier, A., Grellier, A., Guillemot, L. (2014). Aménagement et résilience du commerce urbain en France, L'Harmattan, coll. Itinéraires géographiques, Paris, $269 \mathrm{p}$.

Thill, É. (1966). Les inventeurs du commerce moderne. Des grands magasins aux bébé-requins. Éditions Arthaud, Paris, 317 pages. 


\section{Degré de résilience dans les espaces commerciaux des villes françaises}

(d'un degré de Résilience Commerciale faible de niveau $\mathbf{1}$ (RC1) à un degré de Résilience

Commerciale forte de niveau 4 (RC4)

\section{Résilience commerciale faible 1 ou (RC1)}

\begin{tabular}{|c|c|c|c|}
\hline $\begin{array}{l}\text { Types d'espaces } \\
\text { commerciaux }\end{array}$ & Evolution des commerces & Facteurs de faible résilience & Facteurs de forte résilience \\
\hline Péricentre & $\begin{array}{l}\text { Déclin commercial dans les } \\
\text { années } 1970 \text { et } 1980 \\
\text { La rétraction du centre } \\
\text { commerçant et le déclin du } \\
\text { commerce de proximité } \\
\text { accélèrent le déclin du } \\
\text { péricentre. }\end{array}$ & $\begin{array}{l}\text { Absence de flux } \\
\text { passants importants. } \\
\text { Localisation de services aux } \\
\text { entreprises (agence de } \\
\text { sécurité, interim) et aux } \\
\text { particuliers (assurances) qui } \\
\text { ont pris la place d'anciens } \\
\text { commerces. } \\
\text { Faible attractivité du } \\
\text { commerce péricentral: } \\
\text { éparpillement le long des } \\
\text { rues, faible présence des } \\
\text { enseignes nationales. } \\
\text { Commerce indépendant aux } \\
\text { faibles potentialités } \\
\text { d'évolution. }\end{array}$ & $\begin{array}{l}\text { Faiblesse du prix du foncier et de } \\
\text { l'immobilier commercial. } \\
\text { Seules des opérations d'une certaine } \\
\text { envergure peuvent attirer des } \\
\text { populations extérieures au quartier } \\
\text { (voir RC2). } \\
\text { Renouveau du commerce par le } \\
\text { commerce ethnique dans certains } \\
\text { quartiers. }\end{array}$ \\
\hline
\end{tabular}


Résilience commerciale moyenne mais ponctuelle 2 ou (RC2)

\begin{tabular}{|c|c|c|c|}
\hline $\begin{array}{l}\text { Types d'espaces } \\
\text { commerciaux }\end{array}$ & Evolution des commerces & Facteurs de faible résilience & Facteurs de forte résilience \\
\hline $\begin{array}{l}\text { Grandes } \\
\text { opérations en } \\
\text { péricentral (dont } \\
\text { waterfronts) }\end{array}$ & $\begin{array}{l}\text { Déclin du commerce puis } \\
\text { pratiquement disparition du } \\
\text { commerce de proximité } \\
\text { dans ces espaces } \\
\text { péricentraux depuis les } \\
\text { années 1960-1970 avec le } \\
\text { recul des activités } \\
\text { industrielles } \\
\text { portuaires et le départ des } \\
\text { populations résidantes. }\end{array}$ & 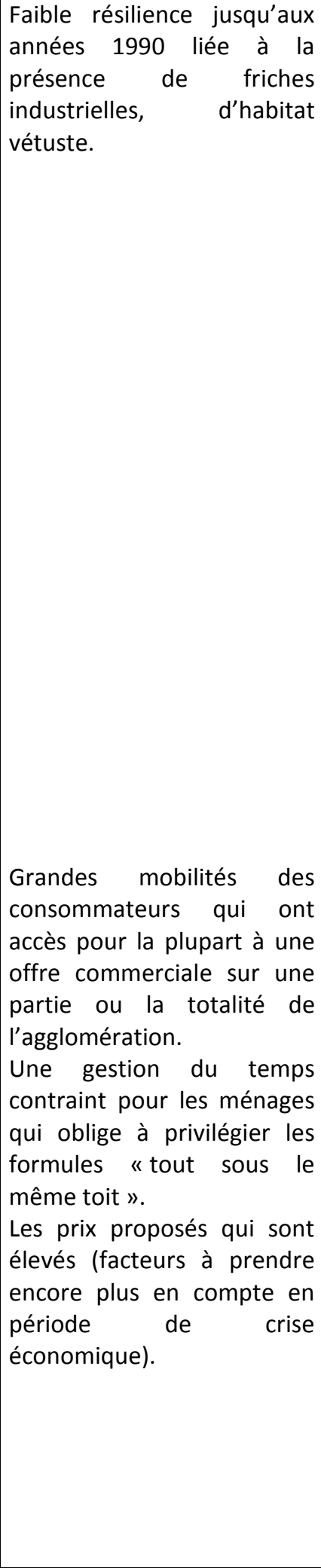 & 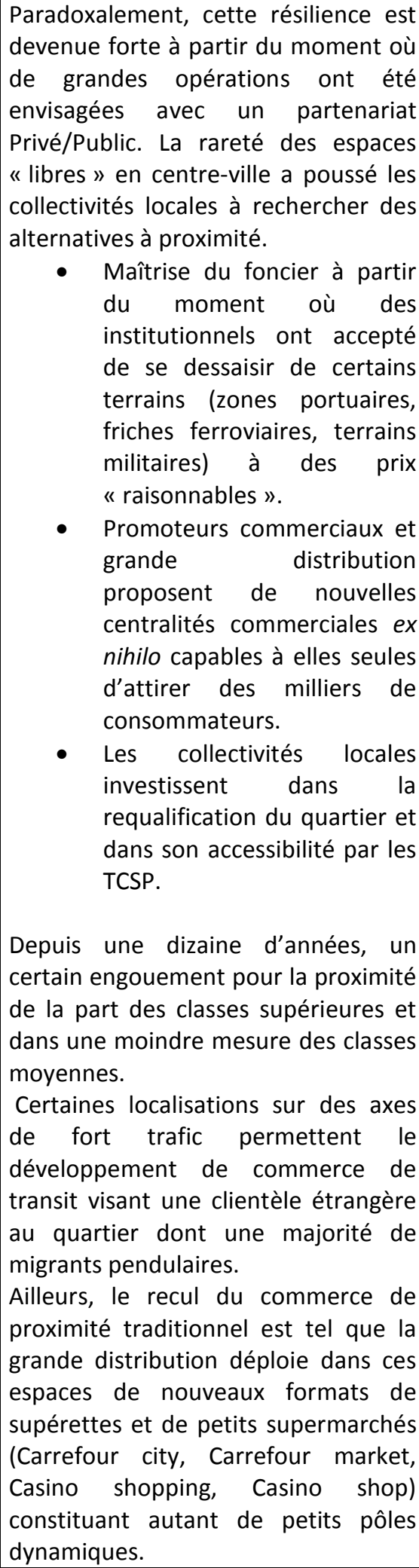 \\
\hline
\end{tabular}




\section{Résilience commerciale forte 3 ou (RC3)}

\begin{tabular}{|c|c|c|c|}
\hline $\begin{array}{l}\text { Types d'espaces } \\
\text { commerciaux }\end{array}$ & Evolution des commerces & Facteurs de faible résilience & Facteurs de forte résilience \\
\hline $\begin{array}{l}\text { Hypercentre des } \\
\underline{\text { villes }}\end{array}$ & $\begin{array}{l}\text { Déclin relatif des centres } \\
\text { commerçants des villes } \\
\text { concurrencés par les } \\
\text { centralités périphériques } \\
\text { ( } 25 \text { à } 30 \% \text { du chiffre } \\
\text { d'affaires du commerce de } \\
\text { l'agglomération contre } 60 \text { à } \\
65 \% \text { pour la périphérie). } \\
\text { Rétraction de ces espaces } \\
\text { centraux dynamiques à } \\
\text { quelques rues principales } \\
\text { animées par un secteur } \\
\text { piétonnier, un centre } \\
\text { commercial intégré ou } \\
\text { l'existence d'un commerce } \\
\text { attractif isolé (Grand } \\
\text { magasin, FNAC). }\end{array}$ & $\begin{array}{l}\text { Accessibilité automobile de } \\
\text { plus en plus difficile (partage } \\
\text { de la chaussée par des TCSP } \\
\text { ou des mobilités douces). } \\
\text { Difficultés de parking. } \\
\text { D'où le risque de perte de } \\
\text { certaines clientèles } \\
\text { motorisées (périphéries et } \\
\text { périurbains non connectés à } \\
\text { des TCSP). }\end{array}$ & $\begin{array}{l}\text { - Image forte du centre-ville } \\
\text { qui n'est pas perçu } \\
\text { seulement comme une } \\
\text { centralité commerciale. } \\
\text { - Attractivité touristique forte } \\
\text { pour certains centres. } \\
\text { - Concentration des enseignes } \\
\text { nationales et internationales } \\
\text { (commerces succursalistes et } \\
\text { franchisés) dans } \\
\text { l'hypercentre. } \\
\text { Animation de l'hypercentre } \\
\text { proche de celles des centres } \\
\text { commerciaux périphériques } \\
\text { (avec Town manager center). } \\
\text { Espace multifonctionnel } \\
\text { (fonction résidentielle, } \\
\text { fonction administrative, } \\
\text { loisirs dont multiplexe, } \\
\text { hôtels-restaurants-café). } \\
\text { Développement } \\
\text { Transports en Commun en } \\
\text { Site Propre (TcSP): Métro, } \\
\text { Tramway ou couloir de bus) } \\
\text { avec des interconnexions qui } \\
\text { privilégient les hypercentres } \\
\text { (pour une certaine clientèle). }\end{array}$ \\
\hline
\end{tabular}


Résilience commerciale très forte 4 ou (RC4)

\begin{tabular}{|c|c|c|c|}
\hline $\begin{array}{l}\text { Types d'espaces } \\
\text { commerciaux }\end{array}$ & Evolution des commerces & Facteurs de faible résilience & Facteurs de forte résilience \\
\hline 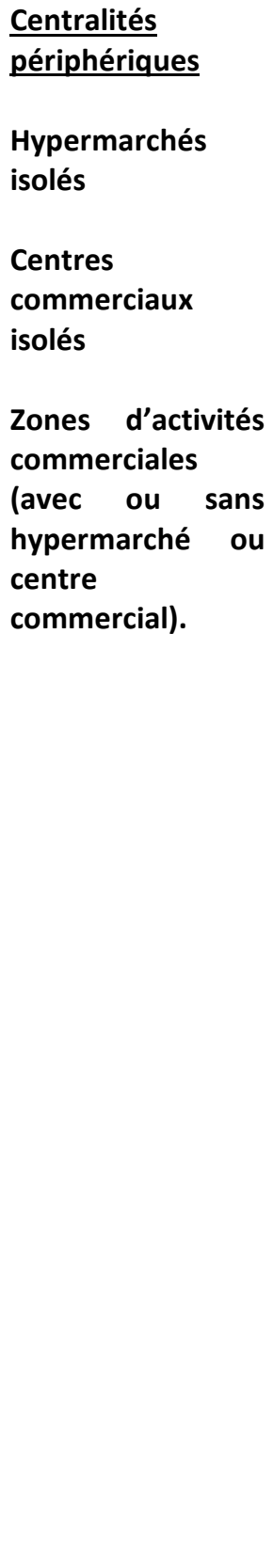 & $\begin{array}{l}\text { Naissance dans les années } \\
\frac{1960-1980}{\text { Saturation du marché }} \\
\text { depuis une dizaine } \\
\text { d'années. } \\
\text { Vieillissement des centres } \\
\text { commerciaux de } \\
\text { premières générations. } \\
\text { Poursuite de la course aux } \\
\mathrm{m}^{2} \text { et apparition des } \\
\text { premiers parcs } \\
\text { commerciaux ou retail } \\
\text { parks. }\end{array}$ & $\begin{array}{l}\text { Pour l'ensemble de ce } \\
\text { commerce périphérique } \\
\text { La saturation du marché avec } \\
\text { une offre pléthorique à un } \\
\text { moment de ralentissement de } \\
\text { la consommation à raviver la } \\
\text { concurrence entre enseignes } \\
\text { (Castorama, Darty en } \\
\text { difficultés), entre espaces } \\
\text { dans une zone commerciale } \\
\text { donnée ou entre zones } \\
\text { commerciales. Les ZAC } \\
\text { excentrées, mal localisées par } \\
\text { rapport aux axes routiers } \\
\text { souffrent. } \\
\text { Les ZAC qui n'ont pas de } \\
\text { locomotives commerciales } \\
\text { (Hypermarché, FNAC, IKEA) } \\
\text { sont moins résilientes. } \\
\text { Résultat: Les premières } \\
\text { friches commerciales } \\
\text { apparaissent. }\end{array}$ & 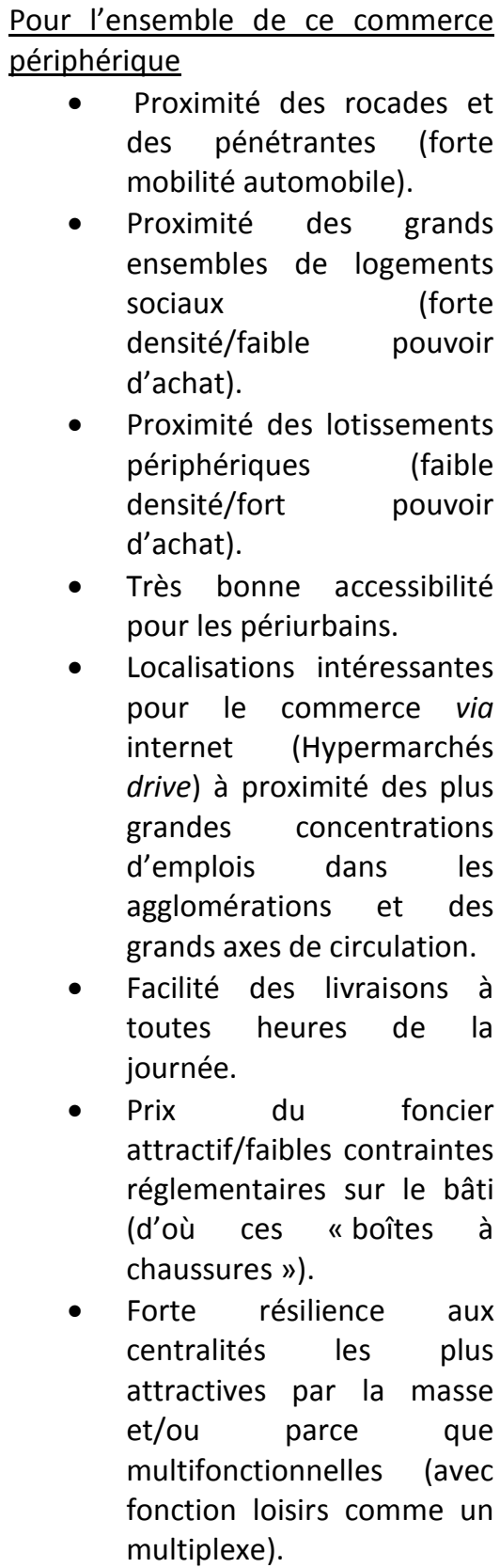 \\
\hline
\end{tabular}

